\title{
Performance Certification of Interconnected Nonlinear Systems using ADMM
}

\author{
Chris Meissen ${ }^{1} \quad$ Laurent Lessard $^{1} \quad$ Murat Arcak $^{2} \quad$ Andrew Packard ${ }^{1}$
}

IEEE Conference on Decision and Control, pp. 5131-5136, 2014

\begin{abstract}
We present a compositional performance certification method for interconnected nonlinear systems, using dissipativity properties of the subsystems along with the interconnection structure. To select the most relevant dissipativity properties, we formulate a large-scale optimization problem, and solve it with the alternating direction method of multipliers (ADMM). The dissipativity properties are allowed to depend on an unknown equilibrium, enabling us to certify performance without explicit knowledge of the equilibrium for the interconnected system. The effectiveness of the algorithm is demonstrated on two examples, including a model of vehicle platoons.
\end{abstract}

\section{Introduction}

In this paper, we consider compositional analysis for performance certification of an interconnection of nonlinear subsystems as depicted in Figure 1. The $G_{i}$ blocks are known subsystems mapping $u_{i} \mapsto y_{i}$, with dynamics described by nonlinear state equations. $M$ is a static matrix characterizing the interconnection topology. The overarching goal of compositional analysis is to establish properties of the interconnected system using only properties of the subsystems along with information about the interaction of the subsystems $(M)$. Henceforth, the term "local" is used to refer to properties or analysis of individual subsystems in isolation. Likewise, "global" refers to the entire interconnected system.

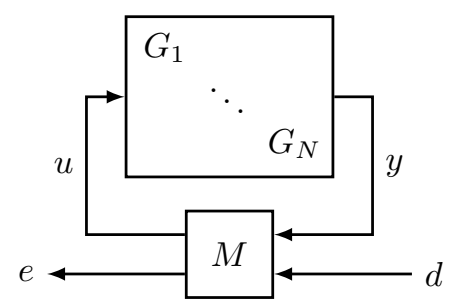

Figure 1: Interconnected system with exogenous input $d$ and performance output $e$.

\footnotetext{
${ }^{1}$ C. Meissen, L. Lessard, and A. Packard are with the Department of Mechanical Engineering at the University of California, Berkeley, CA 94720, USA.

\{cmeissen, lessard, apackard\}@berkeley.edu

${ }^{2} \mathrm{M}$. Arcak is with the Department of Electrical Engineering at the University of California, Berkeley, CA 94720, USA.

arcak@berkeley.edu
}

In this paper, local and global properties are quantified in the framework of dissipative systems [13]; specifically the case with quadratic supply rates [14]. The choice of a supply rate dictates the specific property that is to be verified. For example, different supply rates can be used to verify $L_{2}$-gain properties, passivity, output-strict passivity, etc.

A conventional approach to compositional analysis, as presented for example in $[1,2,10,13]$ is to establish individual supply rates (and storage functions) for which each subsystem is dissipative. Then, a candidate storage function for the interconnected system is sought as a linear combination of the subsystem storage functions.

The method presented here is less conservative in that the local storage functions are treated as decision variables, and are optimized with regards to their particular suitability in certifying desired properties of the interconnected system. Thus, global properties are certified via local certificates that have been automatically generated, as opposed to having been preselected.

The idea of optimizing over the local supply rates (and storage functions) to certify stability of an interconnected system was introduced in [12], with the local supply rates constrained to be diagonally-scaled induced $L_{2}$-norms. This perspective coupled with dual decomposition, gave rise to a distributed optimization problem to certify the stability of the interconnection. More recent work in [9] generalized this in several ways: certifying dissipativity (rather than stability) of the interconnected system with respect to a quadratic supply rate; using arbitrary quadratic supply rates for the local subsystems; and employing ADMM [3] which exposes the distributed certification as a convergent negotiation between local computational agents for each subsystem and a master agent. The roles of the local and master agents are as follows:

- Each local agent receives a proposed supply rate from the master agent and solves an optimization problem to find a supply rate that certifies a local dissipativity property and is close to the proposed supply rate.

- The master agent, with knowledge of the interconnection topology $M$ and the updated supply rates, solves an optimization problem to certify a global dissipativity property and proposes new supply rates to the local agents. 
In this paper, we continue along the direction proposed in [9]. Specifically, we apply this method to nonlinear subsystems using sum-of-squares (SOS) optimization and use the concept of equilibrium-independent dissipativity (EID) for interconnections where the equilibrium point itself is unknown.

\section{Preliminaries}

Dissipativity theory [13]. Consider a time-invariant, continuous-time dynamical system described by

$$
\begin{aligned}
\dot{x}(t) & =f(x(t), u(t)), & & f(0,0)=0 \\
y(t) & =h(x(t), u(t)), & & h(0,0)=0
\end{aligned}
$$

with $x(t) \in \mathbb{R}^{n}, u(t) \in \mathbb{R}^{m}$, and $y(t) \in \mathbb{R}^{p}$. A supply rate is a function $w: \mathbb{R}^{m} \times \mathbb{R}^{p} \rightarrow \mathbb{R}$. A system of the form (1) is dissipative with respect to a supply rate $w$ if there exists a differentiable and nonnegative function $V: \mathbb{R}^{n} \rightarrow \mathbb{R}_{+}$such that

$$
\nabla V(x)^{\top} f(x, u)-w(u, y) \leq 0
$$

for all $x \in \mathbb{R}^{n}, u \in \mathbb{R}^{m}$, and $y=h(x, u)$. Equation (2) is referred to as the Dissipation Inequality Equation (DIE) and $V$ as a storage function.

Equilibrium independent dissipativity [7]. EID can be used to analyze systems where the equilibrium point depends nontrivially on the system input. Consider a system of the form

$$
\begin{aligned}
\dot{x}(t) & =f(x(t), u(t)) \\
y(t) & =h(x(t), u(t))
\end{aligned}
$$

such that for each equilibrium point $x^{*} \in \mathbb{R}^{n}$ there exists a unique $u^{*} \in \mathbb{R}^{m}$ such that $f\left(x^{*}, u^{*}\right)=0$. The equilibrium state-input map is then defined as

$$
k_{u}(x): \mathbb{R}^{n} \rightarrow \mathbb{R}^{m} \text { such that } u^{*}=k_{u}\left(x^{*}\right)
$$

and the equilibrium state-output map is defined as

$k_{y}(x): \mathbb{R}^{n} \rightarrow \mathbb{R}^{p}$ such that $y^{*}=k_{y}\left(x^{*}\right)=h\left(x^{*}, k_{u}\left(x^{*}\right)\right)$

A system of the form (3) is EID with respect to the supply rate, $w$, if there exists a nonnegative storage function, $V: \mathbb{R}^{2 n} \rightarrow \mathbb{R}_{+}$, such that $V\left(x^{*}, x^{*}\right)=0$ and

$$
\nabla_{x} V\left(x, x^{*}\right)^{\top} f(x, u)-w\left(u-u^{*}, y-y^{*}\right) \leq 0
$$

for all equilibrium points $x^{*}$, and for all $x \in \mathbb{R}^{n}$ and $u \in \mathbb{R}^{m}$ where $y=h(x, u), y^{*}=k_{y}\left(x^{*}\right)$, and $u^{*}=k_{u}\left(x^{*}\right)$.

Note that the definition given here is slightly different than in [7] and follows the convention in [4] and [5]. We assume the existence of an equilibrium state-input map whereas in [7] it is assumed that an equilibrium inputstate map exists. Our definition allows systems such as an integrator to be EID.
SOS programming. Let $\mathbb{R}[x]$ be the set of all polynomials in $x$ with real coefficients. $\Sigma[x]:=\{\pi \in \mathbb{R}[x]: \pi=$ $\left.\pi_{1}^{2}+\cdots+\pi_{n}^{2}, \quad \pi_{1}, \ldots, \pi_{n} \in \mathbb{R}[x]\right\}$ is the subset of $\mathbb{R}[x]$ containing the SOS polynomials. A polynomial, $p(x)$, being a sum of squares polynomial is equivalent to the existence of a positive semidefinite matrix $Q$ such that

$$
p(x)=m(x)^{\top} Q m(x)
$$

for a properly chosen vector of monomials, $m(x)$. Therefore checking the nonnegativity of a polynomial can be relaxed to a SOS program and then solved as a semidefinite program (SDP).

Polynomial dynamics. Suppose that $f$ and $h$ in (1) are polynomials. Then, certification of dissipativity of the system with respect to a polynomial supply rate $w$ can be relaxed to a SOS feasibility program:

$$
\begin{aligned}
V(x) & \in \Sigma[x] \\
-\nabla V(x)^{\top} f(x, u)+w(u, y) & \in \Sigma[x, u]
\end{aligned}
$$

Similarly, certification of EID for polynomial systems can be relaxed to a SOS feasibility program [7]:

$$
\begin{aligned}
& V\left(x, x^{*}\right) \in \Sigma\left[x, x^{*}\right] \\
& r\left(x, x^{*}, u, u^{*}\right) \in \mathbb{R}\left[x, u, x^{*}, u^{*}\right] \\
&-\nabla_{x} V\left(x, x^{*}\right)^{\top} f(x, u)+w\left(u-u^{*}, y-y^{*}\right) \\
&+r\left(x, x^{*}, u, u^{*}\right) f\left(x^{*}, u^{*}\right) \in \Sigma\left[x, u, x^{*}, u^{*}\right]
\end{aligned}
$$

Thus, for polynomial dynamics, a storage function that satisfies (2) or (4) can be found by solving (6) or (7), respectively.

Rational polynomial dynamics. Suppose for each state variable $x_{i}$ the associated system dynamics $f_{i}$ are described by rational polynomials of the form

$$
f_{i}(x, u)=\frac{p_{i}(x, u)}{q_{i}(x, u)} \quad \text { for all } i=1, \ldots, n
$$

where $p_{i} \in \mathbb{R}[x, u]$ and $q_{i}-\epsilon \in \Sigma[x, u]$ for some positive $\epsilon$. Then, certifying dissipativity of the system with respect to a polynomial supply rate $w$ can be relaxed to a SOS feasibility program:

$$
\begin{gathered}
V(x) \in \Sigma[x] \\
-\sum_{i=1}^{n} \nabla_{x_{i}} V(x) p_{i}(x, u) \prod_{j \neq i} q_{j}(x, u) \\
+\prod_{i=1}^{n} q_{i}(x, u) w(u, y) \in \Sigma[x, u]
\end{gathered}
$$

Thus, for rational polynomial dynamics, a storage function satisfying (9) can be found by solving (2). Similarly, to the polynomial case, certification of EID for rational polynomial systems can also be formulated as an SOS feasibility program. 


\section{Problem statement}

Consider the interconnected system in Figure 1 which consists of $N$ known subsystems, $G_{i}$, with a known, static interconnection $M \in \mathbb{R}^{m \times p}$. Therefore,

$$
\left[\begin{array}{l}
u \\
e
\end{array}\right]=M\left[\begin{array}{l}
y \\
d
\end{array}\right]
$$

and each $G_{i}$ has dynamics of the form (1) and is characterized by a local state vector $x_{i}$. We assume that the interconnected system is well-posed, meaning that for any $d \in L_{2 e}$, and any initial condition $x_{0}$ there exists unique $e, u, y \in L_{2 e}$ that causally depend on $d$. We assume there exists an equilibrium point $x^{*}$ for which there is a unique $u^{*}=k_{u}\left(x^{*}\right), y^{*}=k_{y}\left(x^{*}\right)$, and $e^{*}=M_{21} y^{*}$.

We assume that the global and local supply rates are quadratic forms. The global supply rate is given by

$$
\left[\begin{array}{c}
d \\
e-e^{*}
\end{array}\right]^{\top} W\left[\begin{array}{c}
d \\
e-e^{*}
\end{array}\right]
$$

and the local supply rates are

$$
\left[\begin{array}{c}
u_{i}-u_{i}^{*} \\
y_{i}-y_{i}^{*}
\end{array}\right]^{\top} X_{i}\left[\begin{array}{c}
u_{i}-u_{i}^{*} \\
y_{i}-y_{i}^{*}
\end{array}\right]
$$

Here, $W$ is give as part of the problem specification, and the $X_{i}$ are real symmetric matrix decision variables. To certify the desired global dissipativity from local EID properties, we pose an optimization problem of the form

$$
\begin{array}{cl}
\underset{X_{1: N}}{\operatorname{minimize}} & 0 \\
\text { subject to } & X_{i} \in \mathcal{L}_{i} \quad \text { for } i=1, \ldots, N \\
& \left(X_{1}, \ldots, X_{N}\right) \in \mathcal{G}
\end{array}
$$

where each $\mathcal{L}_{i}$ constraint is local because it involves only the local supply rate $X_{i}$. The $\mathcal{G}$ constraint is global because it involves all the supply rates.

Before defining the $\mathcal{L}_{i}$ and $\mathcal{G}$ sets, we first introduce the following conformal block partitions

$$
W=\left[\begin{array}{ll}
W_{11} & W_{12} \\
W_{21} & W_{22}
\end{array}\right], \quad X_{i}=\left[\begin{array}{cc}
X_{i}^{11} & X_{i}^{12} \\
X_{i}^{21} & X_{i}^{22}
\end{array}\right]
$$

and the following block-diagonal matrices

$$
X^{j k}=\left[\begin{array}{ccc}
X_{1}^{j k} & & \\
& \ddots & \\
& & X_{N}^{j k}
\end{array}\right] \quad \text { for all } j, k \in\{1,2\}
$$

Recall that $W$ is given while $X_{1}, \ldots, X_{N}$ are to be found. The local and global sets are then defined as follows.

$$
\begin{aligned}
& \mathcal{L}_{i}:=\left\{X_{i} \mid \text { the } i\right. \text {-th subsystem is EID w.r.t } \\
&\text { the supply rate } \left.\left[\begin{array}{l}
u_{i}-u_{i}^{*} \\
y_{i}-y_{i}^{*}
\end{array}\right]^{\top} X_{i}\left[\begin{array}{l}
u_{i}-u_{i}^{*} \\
y_{i}-y_{i}^{*}
\end{array}\right]\right\} \\
& \mathcal{G}:=\left\{X_{1: N} \mid \sum_{i=1}^{N} H_{i} X_{i} H_{i}^{\top}-H_{0} W H_{0}^{\top} \preceq 0\right\}
\end{aligned}
$$

Here, the constant matrices $H_{0}, \ldots, H_{N}$ are defined such that the following identity holds.

$$
\begin{aligned}
& \sum_{i=1}^{N} H_{i} X_{i} H_{i}^{\top}-H_{0} W H_{0}^{\top}= \\
& {\left[\begin{array}{c}
M \\
I
\end{array}\right]^{\top}\left[\begin{array}{cccc}
X^{11} & 0 & X^{12} & 0 \\
0 & -W_{22} & 0 & -W_{21} \\
X^{21} & 0 & X^{22} & 0 \\
0 & -W_{12} & 0 & -W_{11}
\end{array}\right]\left[\begin{array}{c}
M \\
I
\end{array}\right] }
\end{aligned}
$$

In [9] it was shown that if each subsystem is dissipative with respect to a supply rate, $X_{i}$, and that $\left(X_{1}, \ldots, X_{N}\right) \in \mathcal{G}$ then the interconnected system is dissipative with respect to the global supply rate $W$. This result extends directly to the EID case: if the subsystems are EID and the global constraint is satisfied then the interconnected system is dissipative.

The benefit of the formulation in (13) is that the local constraints only depend on the supply rate and storage function for the associated subsystem, so they can be checked separately and in parallel. The global constraint is an LMI that depends on all the supply rates, but does not depend on the storage functions.

Note that our approach seeks a global storage function that is additively separable in the subsystem states. Therefore, it is potentially conservative compared to searching for a storage function that is not separable.

\section{ADMM}

In [9] we demonstrated that ADMM [3] could be used to decompose and solve (13) reliably and efficiently. This method allows us to solve the individual subproblems, which require searching for a potentially high order storage function, in parallel.

ADMM can be used to solve problems of the form

$$
\begin{aligned}
\operatorname{minimize} & f(x)+g(z) \\
\text { subject to } & A x+B z=c
\end{aligned}
$$

where $x$ and $z$ are vector decision variables. ADMM is guaranteed to converge if $f, g$ are closed, proper, and convex, and the Lagrangian has a saddle point [3].

Our problem (13) may be put into the form of (17) by defining the following indicator functions:

$$
\begin{aligned}
\mathbb{I}_{\mathcal{L}_{i}}\left(X_{i}\right) & := \begin{cases}0 & X_{i} \in \mathcal{L}_{i} \\
\infty & \text { otherwise }\end{cases} \\
\mathbb{I}_{\mathcal{G}}\left(X_{1: N}\right) & := \begin{cases}0 & \left(X_{1}, \ldots, X_{N}\right) \in \mathcal{G} \\
\infty & \text { otherwise }\end{cases}
\end{aligned}
$$

Then (13) may be written equivalently as

$$
\begin{aligned}
\underset{X_{1: N}, Z_{1: N}}{\operatorname{minimize}} & \sum_{i=1}^{N} \mathbb{I}_{\mathcal{L}_{i}}\left(X_{i}\right)+\mathbb{I}_{\mathcal{G}}\left(Z_{1}, \ldots, Z_{N}\right) \\
\text { subject to } & X_{i}-Z_{i}=0 \quad \text { for } i=1, \ldots, N
\end{aligned}
$$


so that it is in the canonical form (17). The first term in the objective and the constraints are separable for each subsystem, so the ADMM update takes on the following parallelized form [3].

1. $X$-updates: for each $i$, solve the local problem:

$$
X_{i}^{k+1}=\underset{X \in \mathcal{L}_{i}}{\arg \min }\left\|X-Z_{i}^{k}+U_{i}^{k}\right\|_{F}^{2}
$$

2. $Z$-update: if $\left(X_{1}^{k+1}, \ldots, X_{N}^{k+1}\right) \in G$, then we have found a solution to (13), so terminate the algorithm. Otherwise, solve the global problem:

$$
Z_{1: N}^{k+1}=\underset{Z_{1: N} \in \mathcal{G}}{\arg \min }\left\|\sum_{i=1}^{N}\left(X_{i}^{k+1}-Z_{i}+U_{i}^{k}\right)\right\|_{F}^{2}
$$

3. $U$-update: update $U$ and return to step 1 .

$$
U_{i}^{k+1}=X_{i}^{k+1}-Z^{k+1}+U_{i}^{k}
$$

\section{Examples}

The first example we present demonstrates the scalability and reliability of this approach for certifying performance of large nonlinear systems. The second example is based on the model of vehicle platoons used in [4] and [5].

A large-scale polynomial system The system consists of $N, 2$-state subsystems that share a common dynamical structure, modified from [8], but with different parameters defining each subsystem. An individual subsystem, $H$, has 2 states and is described by

$$
\begin{aligned}
\dot{x}_{1} & =x_{2} \\
H: \quad \dot{x}_{2} & =\frac{-a x_{2}-b x_{1}^{3}+u}{1+c x_{2}^{2}} \\
y & =x_{2}
\end{aligned}
$$

where $a, b, c>0$ are parameters of the subsystem. The positive-definite storage function $V(x):=\frac{a b}{2} x_{1}^{4}+\frac{a c}{2} x_{2}^{4}+$ $a x_{2}^{2}$ and the supply rate $w(u, y):=u^{2}-a^{2} y^{2}$ certify that the $L_{2}$-gain of $H$ is bounded by $a^{-1}$.

Clearly, any well-posed interconnection involving many instances of these systems, along with an interconnection matrix whose spectral norm is less than a results in a dynamical systems whose $L_{2}$ gain is less than 1 . Armed with this insight, the following steps were used to construct examples of large-scale interconnected systems:

1. Choose $\left\{a_{i}, b_{i}, c_{i}\right\}_{i=1}^{N}$ uniformly distributed in $(1,2) \times(0,1) \times(0.5,2)$. These constitute the parameters of system $H_{i}$. Denote $\gamma:=\max _{i} a_{i}^{-1}$.

2. Draw each entry of $S \in \mathbb{R}^{(N+d) \times(N+d)}$ from a standard normal distribution. Overlay a desired sparsity pattern by selectively zeroing out particular entries.
3. Compute $\beta:=\inf _{B} \bar{\sigma}\left(B S B^{-1}\right)$ where $B=$ $\operatorname{diag}\left(b_{1}, \ldots, b_{N}, I_{d}\right), b \in \mathbb{R}_{++}^{N}$. Redefine $S:=\frac{0.99}{\gamma \beta} S$ to ensure that its spectral norm is less than $\gamma$.

4. Choose random nonzero, diagonal scalings $\Psi=$ $\operatorname{diag}\left(\Psi_{1}, \ldots, \Psi_{N}\right)$ and $\Phi=\operatorname{diag}\left(\Phi_{1}, \ldots, \Phi_{N}\right)$.

5. Define $G_{i}:=\Phi_{i} H_{i} \Psi_{i}$, and

$$
M:=\left[\begin{array}{cc}
\Psi^{-1} & 0 \\
0 & I_{d}
\end{array}\right] S\left[\begin{array}{cc}
\Phi^{-1} & 0 \\
0 & I_{d}
\end{array}\right]
$$

Figure 2 below illustrates the interconnection whose (simplicity is now masked by the scalings) that the algorithm must attempt to certify.

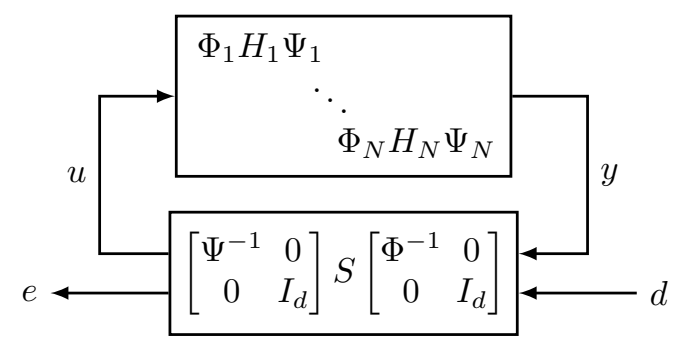

Figure 2: Scaling of the interconnected system of Figure 1 that leaves the closed-loop map unchanged.

We generated 100 random instances of the interconnected system described above, each with $N=100$. The SOS/ADMM algorithm was used to certify the $L_{2}$-gain of the interconnected system is less than or equal to 1 for each instance. Figure 3 shows that the algorithm succeeded for all 100 tests, requiring at most 48 iterations and less than 15 iterations for $90 \%$ of the tests.

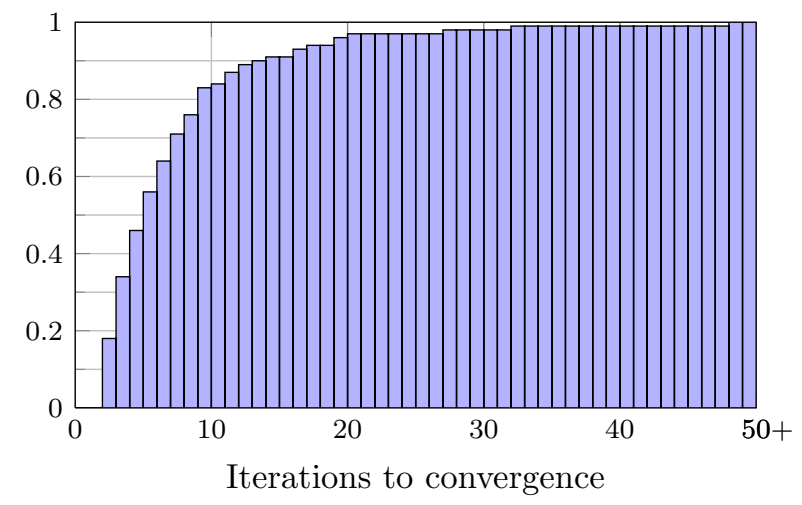

Figure 3: Cumulative plot displaying the fraction of 100 total tests that required at most a given number of iterations to certify the $L_{2}$-gain property of the interconnected system.

Vehicle platooning. In this example we analyze the $L_{2}$-gain properties of a vehicle platoon model. Each vehicle measures its distance to a subset of vehicles (indicated by dashed lines), and adjusts its throttle according 
to some control law. A possible configuration is shown in Figure 4. We would like to certify that under a broad range of control law choices and measurement topologies, bounds on the $L_{2}$-gain properties can be certified. For the purpose of this example, we ignore vehicle collisions.

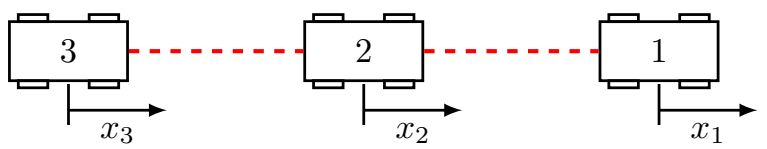

Figure 4: Vehicle platoon example. Each vehicle measures the relative distance of all vehicles connected to it by a dotted line.

Consider $N$ vehicles in a platoon. The dynamics of the $i^{\text {th }}$ vehicle are described by

$$
\begin{aligned}
\Sigma_{i}: \quad \dot{v}_{i} & =-v_{i}+v_{i}^{\text {nom }}+u_{i} \quad i=1, \ldots, N \\
y_{i} & =v_{i}
\end{aligned}
$$

where $v_{i}(t)$ is the vehicle velocity and $v_{i}^{\text {nom }}$ is a nominal velocity. In the absence of a control input $u_{i}(t)$, each vehicle eventually tends to its nominal velocity.

Each vehicle uses the relative distance between itself and a subset of the other vehicles to control its velocity. The subsets are represented by a connected, bidirectional, acyclic graph with $L$ links interconnecting the $N$ vehicles. In Figure 4, the links are shown as dotted lines. Letting $p_{\ell}$ be the relative displacement between the vehicles connected by link $\ell$ then $\dot{p}_{\ell}=v_{i}-v_{j}$ where $x_{i}$ is the leading node and $x_{j}$ is the trailing node. We define $D \in \mathbb{R}^{N \times L}$ as

$$
D_{i \ell}= \begin{cases}1 & \text { if } i \text { is the leading node of edge } \ell \\ -1 & \text { if } i \text { is the trailing node of edge } \ell \\ 0 & \text { otherwise }\end{cases}
$$

Thus, $D$ maps the individual velocities of the vehicles to the relative velocity across each link. That is, $\dot{p}=D^{\top} v$.

Control strategies for velocity regulation of the platoon were presented and analyzed in $[4,5]$. We will consider a general set of control strategies that encompasses those presented in $[4,5]$. Specifically, we will analyze control laws of the form

$$
u_{i}=-\sum_{\ell=1}^{L} D_{i \ell} \phi_{\ell}\left(p_{\ell}\right)
$$

where $\phi_{\ell}: \mathbb{R} \rightarrow \mathbb{R}$ is increasing and surjective, ensuring the existence of an equilibrium point [4]. Defining $\Phi:=\operatorname{diag}\left(\phi_{1}, \ldots, \phi_{L}\right)$, we may represent the system as the block diagram in Figure 5.

The map $\Lambda$ from $\dot{p}$ to $\Phi(p)$, indicated by a dashed box in Figure 5 , is diagonal; each $\dot{p}_{\ell}$ is separately integrated and then the corresponding $\phi_{\ell}$ is applied. Thus, we may write $\Lambda=\operatorname{diag}\left(\Lambda_{1}, \ldots, \Lambda_{L}\right)$, where $\Lambda_{\ell}$ is described by

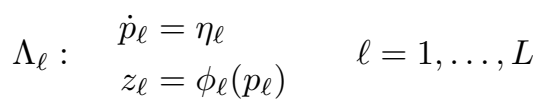

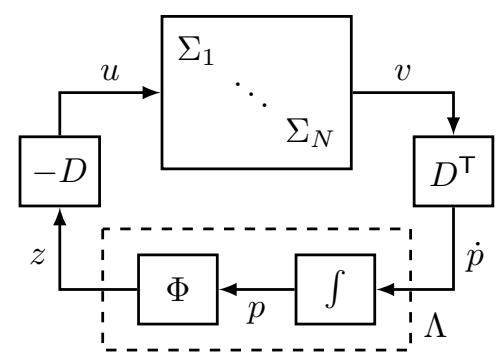

Figure 5: Block diagram of vehicle platoon dynamics.

where $\eta_{\ell}$ is the input and $z_{\ell}$ is the output. The interconnection structure may be transformed into the standard form of Figure 1 by diagonally concatenating $\Sigma:=\operatorname{diag}\left(\Sigma_{1}, \ldots, \Sigma_{N}\right)$ with $\Lambda$. The resulting block diagram is shown in Figure 6.

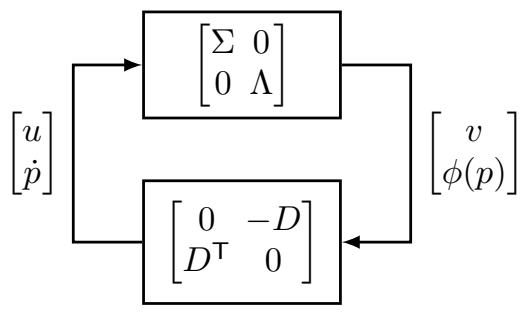

Figure 6: Transformation of Figure 5 into the standard form shown in Figure 1.

Although an equilibrium $\left(v^{*}, p^{*}\right)$ is guaranteed to exist its location depends on the unknown functions $\phi_{\ell}$. Thus, we will exploit the EID properties of the subsystems to establish the desired global property without explicit knowledge of the equilibrium. For each $\Sigma_{i}$ subsystem, we may certify EID by solving an LMI since the dynamics are linear.

For each $\Lambda_{\ell}$ subsystem, a SOS program to certify dissipativity would depend on the choice of the $\phi_{\ell}$ function. However, it is not difficult to show that $\Lambda_{i}$ is EID with respect to the following supply rate (also known as equilibrium independent passivity).

$$
\left[\begin{array}{l}
\eta_{\ell}-\eta_{\ell}^{*} \\
z_{\ell}-z_{\ell}^{*}
\end{array}\right]^{\top}\left[\begin{array}{ll}
0 & 1 \\
1 & 0
\end{array}\right]\left[\begin{array}{l}
\eta_{\ell}-\eta_{\ell}^{*} \\
z_{\ell}-z_{\ell}^{*}
\end{array}\right]
$$

This property can be proven by using the storage function

$$
V_{\ell}\left(p_{\ell}\right)=2 \int_{p_{\ell}^{*}}^{p_{\ell}}\left[\phi_{\ell}(\theta)-\phi_{\ell}\left(p_{\ell}^{*}\right)\right] d \theta
$$

and the property $\left(p_{\ell}-p_{\ell}^{*}\right)\left[\phi_{\ell}\left(p_{\ell}\right)-\phi_{\ell}\left(p_{\ell}^{*}\right)\right] \geq 0$ which follows because $\phi_{\ell}$ is increasing. Therefore, instead of searching over supply rates for the $\Lambda_{\ell}$ subsystems in the ADMM algorithm, we fix (19) as the supply rate. Note that the ADMM algorithm does not require knowledge of the $\phi_{\ell}$ functions or their equilibrium points; it is enough to know the supply rate (19).

For the simulation, we used $N=20$, and each vehicle's nominal velocity was randomly chosen. A linear topology 
was used as in Figure 4. That is, each vehicle measures the distance to the vehicle in front of it and the vehicle behind it. We investigated how a force disturbance applied to the trailing vehicle would affect the velocity of the lead vehicle. Specifically, we augmented the interconnection matrix $M$ (see Figure 1) such that a disturbance $d$ is applied to the last vehicle:

$$
\dot{v}_{N}=-v_{N}+v_{N}^{\text {nom }}+u_{N}+d
$$

and the output $e$ is the velocity of the first vehicle $v_{1}$. We then attempted to certify that the $L_{2}$ gain of the interconnected system from $d$ to $e$ is no greater than $\gamma$ using a global supply rate of the form

$$
\left[\begin{array}{c}
d \\
e-e^{*}
\end{array}\right]^{\top}\left[\begin{array}{cc}
1 & 0 \\
0 & -\gamma^{-2}
\end{array}\right]\left[\begin{array}{c}
d \\
e-e^{*}
\end{array}\right]
$$

Using a bisection search to find the smallest certifiable $\gamma$, we found that $\gamma_{\min } \leq 0.71$. The inequality is due to the fact that our method searches over a restricted class of possible storage functions, and may therefore be conservative. In an effort to bound this conservatism, we performed an ad-hoc search over linear $\phi_{\ell}$ functions, seeking a worst-case $L_{2}$ gain. The result was $\gamma_{\min } \geq 0.49$.

Certain provisions were made to achieve satisfactory convergence of the ADMM routine, and this highlights a potential pitfall of our method that is common to many iterative optimization algorithms. For this example, the local subproblems have the constraints $X_{i}^{11} \geq 0$ for $i=1, \ldots, N$ (these are scalar variables), while the global problem has the constraint $D^{\top} \operatorname{diag}\left(X_{1}^{11}, \ldots, X_{N}^{11}\right) D \leq$ 0 . The only solution that satisfies both of these constraints is $X_{i}^{11}=0$ for all $i$. This further implies that $X_{i}^{12}=X_{i}^{21}=1$ for all $i$. Therefore, even though each of the $\mathcal{G}$ and $\mathcal{L}_{i}$ sets defined in (13) have a nonempty interior, their intersection does not. The result is that the ADMM algorithm oscillates between $X_{i}^{11}>0$ for the local problems and $Z_{i}^{11}<0$ for the global problem, leading to slow convergence and only reaching feasibility (i.e. $\left.X_{i}^{11}=Z_{i}^{11}=0\right)$ in the limit. We addressed this issue by setting $X_{i}^{11}=0$ and $X_{i}^{12}=X_{i}^{21}=1$, effectively removing those variables from the optimization. The feasible region of the resulting problem has a nonempty interior, and the ADMM algorithm converged in a few iterations.

All simulations performed were implemented in MATLAB using the SOSOPT toolbox [11] to solve SOS programs and the CVX toolbox [6] to solve SDP problems.

\section{Acknowledgements}

The authors would like to thank Peter Seiler for helpful discussions.

This work was supported by NASA under Grant No. NRA NNX12AM55A entitled "Analytical Validation Tools for Safety Critical Systems Under Loss-of-Control Conditions", Dr. Christine Belcastro technical monitor.
Any opinions, findings, and conclusions or recommendations expressed in this material are those of the author and do not necessarily reflect the views of NASA.

\section{References}

[1] J. Anderson, A. Teixeira, H. Sandberg, and A. Papachristodoulou. Dynamical system decomposition using dissipation inequalities. In IEEE Conference on Decision and Control, pages 211-216, 2011.

[2] M. Arcak and E. D. Sontag. A passivity-based stability criterion for a class of biochemical reaction networks. Mathematical Biosciences and Engineeering, 5(1):1-19, 2008.

[3] S. Boyd, N. Parikh, E. Chu, B. Peleato, and J. Eckstein. Distributed optimization and statistical learning via the alternating direction method of multipliers. Foundations and Trends in Machine Learning, 3(1):1-122, 2011.

[4] M. Bürger, D. Zelazo, and F. Allgöwer. Duality and network theory in passivity-based cooperative control. In arXiv preprint arXiv:1301.3676, 2013.

[5] S. Coogan and M. Arcak. A dissipativity approach to safety verification for interconnected systems. In IEEE Transactions on Automatic Control, Submitted, 2014.

[6] M. Grant and S. Boyd. CVX: Matlab software for disciplined convex programming, version 2.0 beta. http://cvxr.com/cvx, Sept. 2013.

[7] G. Hines, M. Arcak, and A. Packard. Equilibriumindependent passivity: a new defintion and numerical certification. In Automatica, pages 1949-1956, 2011.

[8] H. Khalil. Nonlinear Systems. Prentice Hall, 3rd edition, 2002.

[9] C. Meissen, L. Lessard, and A. Packard. Performance certification of interconnected systems using decomposition techniques. In American Control Conference, pages 5030-5036, 2014.

[10] N. Sandell, Jr., P. Varaiya, M. Athans, and M. Safonov. Survey of decentralized control methods for large scale systems. IEEE Transactions on Automatic Control, 23(2):108-128, 1978.

[11] P. Seiler. SOSOPT: A toolbox for polynomial optimization. In arXiv preprint arXiv:1308.1889, 2013.

[12] U. Topcu, A. Packard, and R. M. Murray. Compositional stability analysis based on dual decomposition. In IEEE Conference on Decision and Control, pages 1175-1180, 2009.

[13] J. C. Willems. Dissipative dynamical systems part I: General theory. Archive for Rational Mechanics and Analysis, 45(5):321-351, 1972.

[14] J. C. Willems. Dissipative dynamical systems part II: Linear systems with quadratic supply rates. Archive for Rational Mechanics and Analysis, 45(5):352-393, 1972. 